\title{
A New Theory for the Essence and Origin of Electron Spin
}

\author{
Nader Butto \\ Petah Tikva, Israel \\ Email: nader.butto@gmail.com
}

How to cite this paper: Butto, N. (2021) A New Theory for the Essence and Origin of Electron Spin. Journal of High Energy Physics, Gravitation and Cosmology, 7, 1459-1471. https://doi.org/10.4236/jhepgc.2021.74088

Received: August 12, 2021

Accepted: October 17, 2021

Published: October 20, 2021

Copyright $\odot 2021$ by author(s) and Scientific Research Publishing Inc. This work is licensed under the Creative Commons Attribution International License (CC BY 4.0).

http://creativecommons.org/licenses/by/4.0/

\begin{abstract}
Spin is an intrinsic form of angular momentum carried by elementary particles, composite particles, and atomic nuclei. It is wildly believed that spin is a purely quantum mechanical concept and has no classical analogue. In fact, elementary particles are conceived as point objects which have no axis to "spin" around. Therefore, there is no explaining how spin arises at the fundamental level, why particles have the values they do, and what underpins the Pauli Exclusion principle and Bose-Einstein behavior. However, spin is like a vector quantity; it has a definite magnitude, and it has a "direction", in order to spin should be composite. In this paper we propose a physical explanation for spin of the electron at the sub-particle level, relying on the vortex model of the electron. The electron is described as a superfluid frictionless vortex which has a mass, angular momentum and spin to provide a complete explanation of all properties of the electron: it composite, spinning around its own axis, produces a tiny magnetic fields independent of those from its orbital motions. The classical hydrodynamic laws are used to describe the quantum properties of the electron, such as spin, angular momentum, magnetic momentum and a magnetic dipole. The circulation in the vortex is constant, and the angular momentum of the vortex is conserved and has the same value of Planck constant. The direction of the angular momentum of a spinning electron vortex is along the axis of rotation and determined by the direction of spin. The spin quantum number $1 / 2$ has a fixed value which represents the gap between the circulation rate of the core of the vortex and the boundaries of the vortex. The changeable values $+1 / 2$ "spin-up" or $-1 / 2$ "spin-down" indicate the direction of the magnetic dipole of the vortex. The relation between spin and Planck constant is discussed and the origin $\mathrm{h} / 4 \mathrm{pi}$ angular momentum units are revealed.
\end{abstract}

\section{Keywords}

Higgs Field, Irrotational Vortex, Angular Momentum, Magnetic Momentum, 


\section{Introduction}

Spin is an essential property influencing the ordering of electrons and nuclei in atoms and molecules, giving it great physical significance in chemistry and solid-state physics. Spin is likewise an essential consideration in all interactions among subatomic particles, whether in high-energy particle beams, low-temperature fluids or the tenuous flow of particles from the sun known as the solar wind.

Indeed, many if not most physical processes, ranging from the smallest nuclear scales to the largest astrophysical distances, depend greatly on interactions of subatomic particles and the spins of those particles. It is physically manifest in multiple phenomena such as Pauli pairs, photon polarisation, selective spin of neutrino species, superconductivity, entanglement and electron spin, g-factor [1].

Spin magnetic moments create a basis for one of the most important principles in chemistry. The theory plays further roles than just the explanations of doublets within electromagnetic spectrum. This additional quantum number, spin, became the basis for the modern standard model used today, which includes the use of Hund's rules, and an explanation of beta decay.

Spin was originally conceived as the rotation of a particle around some axis. However, at the current level of understanding, the electron like other elementary particles such as quarks, and bosons (such as the photon) are all imagined as point-like, and are not solid "objects" that can "rotate" in space, therefore, it is generally accepted that there is no explanation, that the properties are merely intrinsic, that QM is complete as a theory as it is, e.g. [2].

The discovery of electron spin goes back to 1920s, by Otto Stern and Walther Gerlach of the University of Hamburg in Germany during a series of important atomic beam experiments. Knowing that all moving charges produce magnetic fields, they proposed to measure the magnetic fields produced by the electrons orbiting nuclei in atoms. They designed an experiment, which was done with silver that was put in an oven and vaporized. The basic observation is that a stream of quantum particles can be sent across a magnetic field that is directed perpendicular to the stream.

To their surprise, the result was that silver atoms formed a beam that passed through a magnetic field was observed to possess two possible discrete angular momenta despite having no orbital angular momentum [3].

The screen reveals discrete points of accumulation, rather than a continuous distribution [4], owing to their quantized spin.

The results show that particles possess an intrinsic angular momentum that is closely analogous to the angular momentum of a classically spinning object, but that takes only certain quantized values. If the particles deflect, then you can say 
they have spin. If they don't, you say they have spin zero.

Wolfgang Pauli in 1924 was the first to propose a doubling of electron states due to a two-valued non-classical "hidden rotation" [5].

And in 1927 he worked out the mathematical theory in depth. When Paul Dirac derived his relativistic quantum mechanics in 1928, electron spin was an essential part of it and it predicted the discovery of the positron [6] and correctly gave the value of the spin, $\mathrm{h} / 4 \mathrm{pi}$ angular momentum units.

In quantum mechanics and particle physics, a spin is an intrinsic form of angular momentum carried by elementary particles, composite particles (hadrons), and atomic nuclei [7] [8].

Spin is one of two types of angular momentum in quantum mechanics, the other being orbital angular momentum.

In 1925, George Uhlenbeck and Samuel Goudsmit at Leiden University suggested the simple physical interpretation of a particle spinning around its own axis and the term "spin", however, still remains.

The spinning electrons produce tiny magnetic fields independent of those from their orbital motions. Spin is like a vector quantity; it has a definite magnitude, and it has a "direction". All electrons have the same magnitude of spin angular momentum, which is indicated by assigning the particle a spin quantum number.

In addition, spin is quantized, meaning that only certain discrete spins are allowed it never changes, and has only two possible orientations: in electron for example could be $+1 / 2$ and $-1 / 2$. Since the sign indicates the direction, we tend to call these "spin-up" and "spin-down."

Massless particles are a bit different, they don't have a rest frame, instead they have a different quantity that which is helicity. This too is quantized in half integer units of h-bar. Helicity behaves similarly to spin and it is for this reason that the photon is said to have spin 1, but there are real differences that shouldn't be overlooked.

The direction of the angular momentum of a spinning object is along the axis of rotation and determined by the direction of spin. However, electrons are not literally spinning balls of charge, but they do have intrinsic angular momentum.

Despite the fact that there is a mathematical description of how it behaves, in particular, of how the total spin of a system of particles depends on the spins of the constituents, the origin and the essence of the spin are not known and till today there is no real deep understanding of what spin is, much beyond the fact that it is an attribute of an elementary particle.

If the electron considering as a spinning ball with the known sizes, the surfaces of the charged electron would have to be moving faster than the speed of light in order to produce the measured magnetic moments. In addition, rotating the spin of a spin-1/2 particle by 360 degrees doesn't get you exactly the state you started with.

Furthermore, there is no explaining how spin arises at the fundamental level, why particles have the values they do, and what underpins the Pauli Exclusion 
principle and Bose-Einstein behavior.

Therefore, quantum mechanics, does not offer any deeper explanation of why spin numbers prevent fermions from co-locating. Why should spin be exclusively $1 / 2$ for elementary fermions, yet merely predominately 1 for bosons? What is the basis of this differentiation?

These situations create all sorts of complications that make spin one of the more challenging aspects of quantum mechanics.

In this paper, based on vortex structure of the electron, the nature and origin of the its spin is discussed, the origin of $1 / 2$ of elementary fermion is explained, in addition, the relation between spin and Planck constant is discussed.

\section{The Origin of the Spin Angular Momentum}

Although the question of whether elementary particles actually rotate is ambiguous, the rotating particle picture is correct insofar as spin obeys the same mathematical laws as quantized angular momentum do, in particular, spin implies that the particle's phase changes with angle.

The angular momentum of the electron indicates that there is an internal rotation (spin) that confers upon it its rest mass. According to Higgs theory, the interaction between particles and the Higgs field is continuously maintained and renewed, converting the amorphous potential energy of the field into individual structures. The seemingly empty space that surrounds the electron is teeming with pairs of particles and antiparticles that fleet in and out of existence to create "virtual particles" cloud.

Although precisely measuring this cloud is beyond the capabilities of modern methods, the current model predicts that electrons are slightly aspheric, with a distortion characterized by the electric dipole moment. However, no experiment so far has detected this deviation [9].

In previous article, the electron was presented as a classical vortex therefore the spin could be explained in classical terms [10].

The proposed idea is that the electrons are irrotational vortices of frictionless superfluid space with concentric streamlines that were created from the primordial vacuum during the Big Bang.

The superfluid accommodates the rotation by forming a lattice of quantized vortices in which the vortex core, typically singular, breaks the topological constraint against rotational motion.

The superfluid vortices are frictionless, have a mass, angular momentum and spin that can be studied applying hydrodynamic laws. This allows us to compare spin's behavior to the behavior of other liquid or gas vortices that we feel we understand better.

Maxwell worked out a theory of electromagnetism assuming that every magnetic tube of force was a vortex with an axis of rotation coinciding with the direction of the force. Several properties have been mathematically proved for a perfect frictionless fluid. 
Therefore, in order to understand the law that governs the electron structure and behavior we should take into consideration the fluid dynamics of the vortices. To do so we can apply Helmholtz's three theorems which are as follows [11].

The strength of a vortex filament is constant along its length.

1) A vortex filament cannot end in a fluid; it must extend to the boundaries of the fluid or form a closed path.

2) In the absence of rotational external forces, a fluid that is initially irrotational remains irrotational.

The hydrodynamic laws are applied to describe the particle properties in the quantum scale domain.

Vortex is a mass of fluid in whirling or rotary motion in the absence of friction would make it impossible to destroy vortex motion. This solution relies on the current standard theory assumption that particle's spin is the result of an initial impulse generated in the Big Bang conserved over billions of years of evolution in a frictionless environment.

Motion in such a fluid would be a permanent flow pattern; the velocity of the fluid element instantaneously passing through a given point in space would be constant in time, this is an irrotational vortex.

Therefore, there are two kinds of angular momentum of the electron: The spin angular momentum and orbital angular momentum. The spin angular momentum is the angular momentum about the object's centre of mass, while the orbital angular momentum is the angular momentum about a chosen center of rotation.

Angular momentum is a form of inertia, reflecting the object's size, shape, mass, and rotational velocity. It is typically represented as a vector $(\mathrm{L})$ pointing along the axis of rotation.

\section{The Origin of Spin 1/2 Particle}

All particles can spin, like the planet on its axis, however, it would be possible in theory to change the speed or direction of the planet spin. With particles, spin has a fixed value that depends only on the type of particle. Once it was formed it will remain very stable and their decay very slow due to the very low viscous forces of the adjacent vacuum. Thus spin is quantized, meaning that only certain discrete spins in which the vortex is stable are allowed.

The angular momentum around an axis is the generator of rotations about that axis, and its value tells us the rate at which the wave function changes as it's rotated about that axis, and only a discrete set of rates are possible since we have to get the original wave function back after the boundary rotation of the vortex by $2 \pi$ radians or 360 degrees.

In order to maintain stable rotation the vortex should complete $2 \pi r, 360$ degrees, which allows the minimal time needed to exist $t=2 \pi r / c$.

At the moment of formation of the particle, a gap remains between the core rotation and the boundaries speed rotation. The core could make a complete rotation then the streamlines follow this rotation with some retardation. This is 
actually the reason for the curvature of the streamlines.

Indeed, during the vortex formation, the different angular speed creates differential rotation so virtual photons closer to the center complete greater fraction of orbit in a given time (Figure 1).

The spin is the rate of rotation between the boundaries and the center of the vortex. When the center complete two orbital rotation in relation to the boundaries the spin is considered to be $1 / 2$. Similar behavior is observed in different spiral galaxies.

Hence the magnitude of the spin quantum number is an intrinsic attribute of a particle related to the relation between the numbers of torque of the core of the vortex to the numbers of torque in the external part. A spin $1 / 2$ particle needs two full rotations $\left(2 \times 360^{\circ}=720^{\circ}\right)$ of the core until it is again in the same state of complete rotation in the boundaries of the vortex.

\section{Relation between Angular Momentum and the Reduced Planck Constant}

Niels Bohr (1913) found that the Plank constant could explain the energy structure of electrons in the atom [12].

Electron spin is the particles' intrinsic angular momentum $(S)$ quantified by the equation

$$
S=\hbar \sqrt{S+1},
$$

where $s=n / 2$ and $n$ is any non-negative integer and is described in units $\mathrm{N} \cdot \mathrm{m} \cdot \mathrm{s}$, or $\mathrm{kg} \cdot \mathrm{m}^{2} \cdot \mathrm{s}^{-1}$.

In hydrodynamics, the velocity of the fluid element instantaneously passing through a given point in space in the vortex with radius $r$ is constant in time; therefore, the circulation or the vorticity $\Gamma=2 \pi r c$ is constant. $\Gamma_{m}$ is a conserved momentum; therefore, $2 \pi \mathrm{rcm}$ is constant, which corresponds to the Planck constant.

Thus,

$$
\Gamma_{e}=2 \pi r_{e} c, h=\Gamma_{e} m_{e},
$$

and

$$
\Gamma_{e}=h / m_{e}=\text { constant }
$$

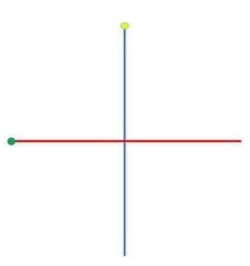

0 degrees 90180 degrees

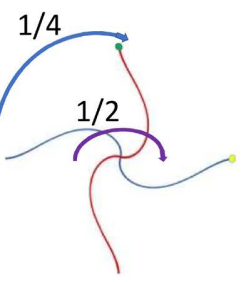

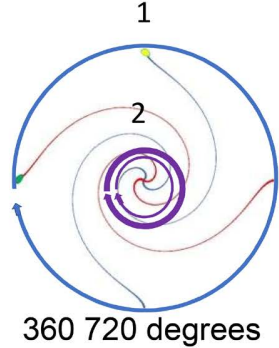

Figure 1. The differential rotation is due to a greater fraction of their orbit in a given time, when the boundaries complete one circle of 360 degrees the center complete two circles of 720 degrees. 
Therefore, the Plank constant is the expression of the angular momentum of the electron vortex which describes the angular momentum of the internal energetic filaments which are rotating with angular velocity and angular frequency typically measured in cycles or rotations per unit time measured with units of radian per second. One radian is the angle subtended at the center of a circle by an arc that is equal in length to the radius of the circle.

The length $S$ of the enclosed arc is equal to the radius multiplied by the magnitude of the angle in radians is given by,

$$
S=\theta r
$$

where $S$ is arc length, $r$ is the radius of the circle, $\theta$ is the subtended angle enclosed by the arc in radians. The magnitude in radians of such a subtended angle is equal to the ratio of the arc length to the radius of the circle; that is,

$$
\underline{\theta}=S / r
$$

It follows that the magnitude in radians of one complete revolution (360 degrees) is the length of the entire circumference divided by the radius, or $2 \pi r / r$, or $2 \pi$. Thus $2 \pi$ radians is equal to 360 degrees, meaning that one radian is equal to $180 / \pi$ degrees. The angular momentum of one radian is a minimal discrete value which is equal to the Planck constant divided by (or reduced by) $2 \pi$, and denoted $\hbar$ ("h-bar"). However, angular momentum is a description of particle spin. Therefore, the final angular momentum is a multiple of the spin value which is $1 / 2$ times the vortex angular momentum to restore the original state. The final angular momentum will be expressed as Planck's constant divided by $2 \pi \times 1 / 2=$ $4 \pi$.

The SI unit of spin is $\left(\mathrm{kg} \cdot \mathrm{m}^{2} \cdot \mathrm{s}^{-1}\right)$, just as with classical angular momentum indicating that Planck constant is an expression of classical angular momentum.

\section{The Formation of Magnetic Field}

In the irrotational vortex, the rates of rotation of the fluid in the vortex is greatest at the center and decreases progressively with distance from the center until there is no gradient pressure on the boundaries of the vortex where the flow is laminar and the friction is null.

In the electron vortex, the flow of Higgs particles that moves in spirals downward in the funnel creates a pressure gradient normal to the vortex center and acts along the central axis of vortex spin. If the velocity of the space circulation reaches the limiting speed, $c$, which is the speed of light in the absolute vacuum, and the velocity field gradient around the core of the vortex becomes the postulated limiting angular rotation, $\omega$, the space breaks down, creating a spherical void, which is defined as a field-less, energy-less, and space-less volume of vacuum at the vortex center.

It follows moving down in the center and around the spiral and returns from the upper side of the central axis generating the magnetic momentum and magnetic dipole (Figure 2). 
From classical electrodynamics, a rotating electrically charged body creates a magnetic dipole with magnetic poles of equal magnitude but opposite polarity.

A spinning system along an axis with angular momentum has a torque when the force is directed toward the center of gravity; known as the Coriolis effect, and when the boundaries of the vortex complete on cycle the magnetic field rotates making the whole structure rotate as a rigid sphere acting on the vortex to maintain its rotation as one unit and the spiral arm curl is fixed for all time (Figure 3 ).

One consequence is that an external magnetic field exerts a torque on the electron magnetic moment and the vortex creates the magnetic field and the magnetic field maintain the rotation of the vortex.

For magnetic dipoles, the dipole moment points from the magnetic south to the magnetic north pole. The electron exists in a magnetic field which exerts a

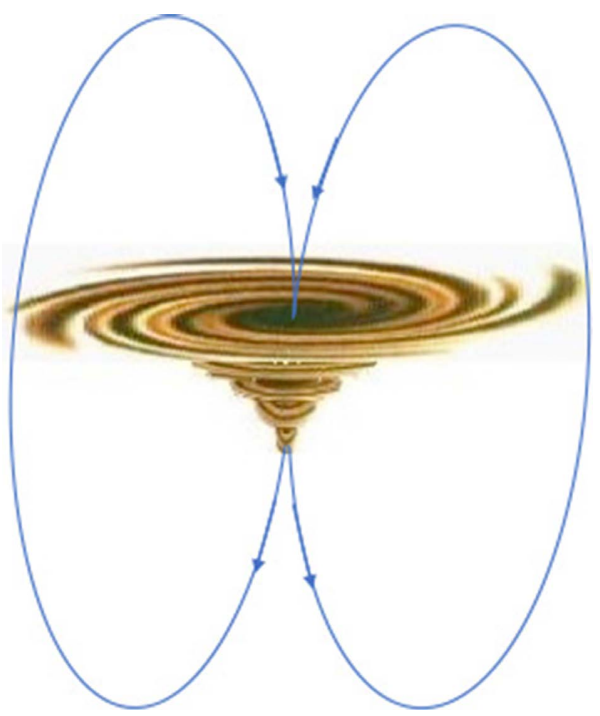

Figure 2. The charge flowing through a circular torque experienced by a vortex forms magnetic energy who's field lines are perpendicular to the direction of the charge rotation.

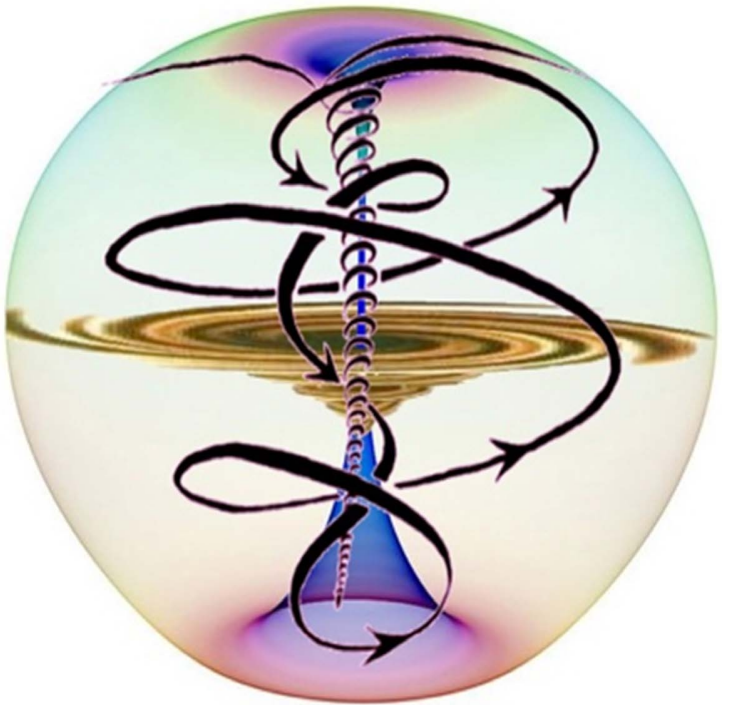

Figure 3. Artistic presentation of the rotating egg shape magnetic field around the electron. 
torque opposing its alignment creating a potential energy that depends on its orientation with respect to the field.

The electron can have a magnetic dipole is oriented in a way that the negative pole is up and the positive pole down. In this case, the projection value said to be $-1 / 2$, while in the opposite direction said to be $+1 / 2$ (Figure 4 ).

The magnetic field separates the vortex from the adjacent vacuum and converts it in independent entity with perpetual mobile. The projection can be changed, but the total spin of $1 / 2$ related to the spiral arm curl is fixed for all time.

If the electron is visualized as a classical charged particle rotating about an axis with angular momentum $L$, its magnetic dipole moment $\mu$ is given by:

$$
\mu=-q g L / 2 m_{e}
$$

where $q$ is the electron charge, $g$ is the g-factor of the electron, $L$ is the angular momentum and $m_{e}$ is the electron rest mass.

The magnetic energy of an electron is approximately twice what it should be in classical mechanics. The factor of two multiplying the electron spin angular momentum comes from the fact that it is twice as effective in producing magnetic moment. This factor is called the electronic spin $g$-factor.

The $g$-factor is an essential value related to the magnetic moment of the subatomic particles and corrects for the precession of the angular momentum.

The intrinsic magnetic moment $\mu$ of a particle with charge $q$, mass $m$, and spin $s$, is

$$
\mu=g(q / 2 m) s
$$

where the dimensionless quantity $g$ is called the $g$-factor. The $g$-factor is a part of the calculation for the gyromagnetic ratio, which is the ratio of the magnetic moment of a particle to the angular momentum of the same particle. The spin $g$-factor is approximately two. The reason of this has been a source of mystery ever since spin was discovered. The vortex structure of the electron help solve this mystery.

\section{The Spin Angular Momentum and Magnetic Momentum}

Angular momentum can be considered a rotational analog of linear momentum.
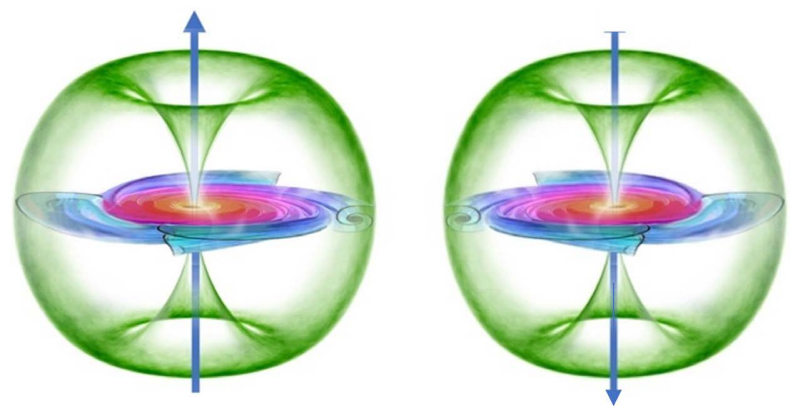

Figure 4. Two opposite spins the one has projection value said to be up with spin up (left) $+1 / 2$ and spin down (right) $-1 / 2$. 
Thus, where linear momentum $p$ is proportional to mass $m$ and linear speed $V$,

$$
p=m v
$$

The magnitude of the angular momentum of the vortex $L$ is proportional to moment of inertia $I$ and angular speed $\omega$ measured in radians per second.

$$
L=I \omega
$$

$L$ should be referred to as the angular momentum relative to that center.

Because $I=r^{2} m$ for a single particle and $\omega=c / r$ for circular motion, angular momentum can be expanded, $L=r^{2} m c / r$ and reduced to,

$$
L=r m_{e} C
$$

where $r$ is the radius of the vortex $m_{e}$ is the electron rest mass and $c$ is the speed of light.

A spin magnetic moment is the magnetic moment caused by the spin of the charged electron vortex. The magnitude of the magnetic dipole moment of the vortex is:

$$
\mu=I A \text {, }
$$

where $I$ is the current of the charge inside the vortex and $A$ is the area of the vortex of both sides which is $2 \pi r^{2}$ where $r$ is the radius of the vortex.

The current inside the vortex passes by a particular point on the vortex in a certain time, so we can calculate a current

$$
I=q / T \text {. }
$$

where $q$ is the magnitude of the electron charge and $T$ is its cycle period of the vortex [13].

The electron as a vortex has a minimum time less than which the electron converts into a virtual particle. If we assume that the electron vortex has a circular shape that needed to complete one rotation cycle $2 \pi r_{e}$, the orbital period will be:

$$
T=2 \pi r / v,
$$

where $r$ is the radius of the core of electron vortex and $v$ is the rotation speed of the electron vortex.

The area of the vortex is approximately a circle, however, the surface depression in the center double the surface area, therefore,

$$
A=2 \pi r^{2}
$$

then the magnitude of the magnetic dipole moment will be

$$
\mu=I A=q v 2 \pi r^{2} /(2 \pi r)=q c r .
$$

where $q$ is the electron charge.

The ratio between magnetic momentum and angular momentum

$$
\mu / L=q c r / r m_{e} c=q / m_{e}
$$

Therefore

$$
\mu=\left(q / m_{e}\right) L
$$


Thus, the electron is visualized as a vortex charged particle rotating about an axis with angular momentum $L$, its magnetic dipole moment $\mu$ in full vector form, is written as

$$
\boldsymbol{\mu}=-\left(q / m_{e}\right) \boldsymbol{L}
$$

The negative sign appears because the electron has a negative charge.

Therefore there is no need to add the mysterious g-factor in order to obtain the experimental results.

If the angular momentum of one radian is a minimal discrete value which is equal to the Planck constant reduced by $2 \pi$. Bohr magneton $\mu_{B}$ express the magnetic moment of one vortex radiant in terms of the reduced Planck constant $\hbar$.

$$
\mu_{B}=e \hbar / 2 m_{e}
$$

Since the magnetic moment is quantized in units of $\mu_{B}$, correspondingly the angular momentum is quantized in units of $\hbar$.

\section{Conclusions}

The electron quantum properties can be accurately described using classical physics and hydrodynamics laws.

Most individual particles and combinations of particles produce a magnetic field very much like that of a spinning vortex of charge. Experiments and observations showed that this spin is most easily described as an angular momentum. In the vortex, spin acts like angular momentum because it is related to the spiral arm rotation, so everything we know about angular momentum is also true of spin.

Hydrodynamic laws are applied to study the behavior of the superfluid vortex of the electron which has internal rotation, and angular momentum, and spin, which confers upon it its rest mass.

The electron is a cloud, a composite web and energy motion processes which has irrotational vortex, created during the big bang.

The angular momentum of a vortex filament of the particle is the sum of angular momenta of the individual Higgs particles which behave as a fluid. In this way, angular momentum is sometimes described as the rotational analog of linear momentum.

The $1 / 2$ spin is an expression of the gap between the rotation rate of the vortex core which is double rotation rate of the vortex boundaries. This unchangeable gab is fixed at the moment of electron formation during the big bang. The $-1 / 2$ and $+1 / 2$ are indication of the orientation of the magnetic dipole like south and north.

The conserved angular momentum of the electron is related to constancy of circulation or vorticity in the irrotational vortex multiplied by its mass which has the same value of Planck constant. Planck constant expresses the angular momentum of $2 \pi$ radians which is equal to 360 degrees. The minimal discrete value 
which is equal to the Planck constant reduced by $2 \pi$ radians denoted as $\hbar$ ("h-bar").

The angular momentum of one radian is the minimal discrete Bohr magneton $\mu_{B}$ expresses the magnetic moment of one vortex radiant in terms of the reduced Planck constant $\hbar$.

Since the magnetic moment is quantized in units of $\mu_{B}$, correspondingly the angular momentum is quantized in units of $\hbar$.

The final angular momentum of the electron is a multiple of the spin value which is $1 / 2$ times the vortex angular momentum, which might be expressed as Planck's constant divided by 4 pi.

The origin of spin completes the properties of the electron. All properties such particle wave duality [14], electron charge, mass, spin angular momentum and magnetic momentum, can now be derived from the vortex structure and match all experimental observations of the electron. The hydrodynamic process of the vortex may open new horizons to understand the properties and behavior of fermions such as quarks and the internal structure of the proton and neutrons, and to understand the hydrodynamic process of interaction with large scale spiral shock fronts that might be responsible for the observed rotational properties of giant molecular clouds in galactic disk, and better describe the formation and structure of galaxies, supernovas, and other astrophysical systems such as black holes, their plasma dynamics and electromagnetic fields giving a new model for unification of the four forces and the basis for the theory of everything.

\section{Acknowledgements}

This research did not receive any specific grant from funding agencies in the public, commercial, or not-for-profit sectors.

\section{Conflicts of Interest}

The author declares no conflicts of interest regarding the publication of this paper.

\section{References}

[1] Dirac, P.A.M. (1942) Bakerian Lecture-The Physical Interpretation of Quantum Mechanics. Proceedings of the Royal Society A, 180, 1-40. https://doi.org/10.1098/rspa.1942.0023

[2] Colbeck, R. and Renner, R. (2011) No Extension of Quantum Theory Can Have Improved Predictive Power. Nature Communications, 2, Article No. 411. https://doi.org/10.1038/ncomms1416

[3] Eisberg, R. and Resnic, R. (1985) Quantum Physics of Atoms, Molecules, Solids, Nuclei, and Particles. John Wiley \& Sons, Hoboken, 272-273.

[4] Gerlach, W. and Stern, O. (1922) Der experimentelle Nachweis der Richtungsquantelung im Magnetfeld. Zeitschrift für Physik, 9, 349-352. https://doi.org/10.1007/BF01326983

[5] Abraham, P. (1991) Niels Bohr's Times. Clarendon Press, Oxford, p. 201. 
[6] Anderson, C.D. (1933) The Positive Electron. The Physical Review, 43, 491. https://doi.org/10.1103/PhysRev.43.491

[7] Merzbacher, E. (1998) Quantum Mechanics. 3rd Edition. University of North Carolina, Chapel Hill, pp. 372-373.

[8] Griffiths, D. (2005) Introduction to Quantum Mechanics. 2nd Edition. Pearson Prentice Hall, Hoboken, pp. 183-184.

[9] Hudson, J.J., Kara, D.F.M., Smallman, I.J., Sauer, B.E., Tarbutt, M.R. and Hinds, E.A. (2011) Improved Measurement of the Shape of the Electron. Nature, 473, 493-496. https://doi.org/10.1038/nature10104

[10] Butto, N. (2020) Electron Shape and Structure: A New Vortex Theory. Journal of High Energy Physics, Gravitation and Cosmology, 6, 340-352. https://doi.org/10.4236/jhepgc.2020.63027

[11] Kuethe, A.M. and Schetzer, J.D. (1959) Foundations of Aerodynamics. 2nd Edition. John Wiley \& Sons, Inc., New York.

[12] David, J. (1995) Griffiths, Introduction to Quantum Mechanics. Prentice Hall, Hoboken, p. 155.

[13] Butto, N. (2021) A New Theory for the Essence and Nature of Electron Charge. Journal of High Energy Physics, Gravitation and Cosmology, 7, 1190-1201. https://doi.org/10.4236/jhepgc.2021.73070

[14] Butto, N. (2020) A New Theory on Electron Wave-Particle Duality. Journal of High Energy Physics, Gravitation and Cosmology, 6, 567-578. https://doi.org/10.4236/jhepgc.2020.64038 